\title{
miR-106b-5p promotes stem cell-like properties of hepatocellular carcinoma cells by targeting PTEN via PI3K/Akt pathway
}

This article was published in the following Dove Press journal:

OncoTargets and Therapy

\section{Dong-Min Shi* \\ Xin-Yu Bian* \\ Cheng-Dong Qin* \\ Wei-Zhong Wu}

Liver Cancer Institute, Zhongshan Hospital, Fudan University, Key Laboratory of Carcinogenesis and Cancer Invasion, Ministry of Education, Shanghai, People's Republic of China

*These authors contributed equally to this work
Correspondence: Wei-Zhong Wu Liver Cancer Institute, Zhongshan Hospital, Fudan University, Key Laboratory of Carcinogenesis and Cancer Invasion, Ministry of Education, I80 Fenglin Road, Shanghai 200032, People's Republic of China

Tel +862160268628

Fax +862164037181

Email drwzwu2015@163.com
Background: The miRNA miR-106b-5p has been previously reported to be increased in hepatocellular carcinoma (HCC) tissues compared to cirrhotic tissues. The purpose of this study was to detect its expression in HCC cell lines with distinct metastatic potentials and to explore the molecular mechanisms underlying HCC stemness and migration.

Methods: miR-106b-5p expression was studied in HCC tissues and cell lines. In vitro cancer stem cell (CSC)-like properties, cell migration and invasion were compared between HCC cell lines with upregulation or downregulation of miR-106b-5p. In vivo tail vein injection models were established to evaluate the role of miR-106b-5p in lung metastasis. Bioinformatics programs, luciferase reporter assay and rescue experiments were used to validate the downstream targets of miR-106b-5p. The relationship between the expression of the targeted gene and clinicopathological parameters was also analyzed.

Results: miR-106b-5p expression was higher in HCC tissues and cell lines than that in nontumor tissues and hepatocyte Chang liver, respectively. Upregulation of miR-106b-5p exhibited a promoting role in CSC properties, cell migration and activation of phosphatidylinositol-3 kinase $(\mathrm{PI} 3 \mathrm{~K}) /$ Akt signaling in vitro, as well as in lung metastasis in vivo. However, downregulation of miR-106b-5p exhibited the opposite effect. Furthermore, PTEN was verified as a direct target of miR-106b-5p. Upon clinicopathological analysis, lower level of PTEN was significantly associated with more aggressive characteristics. Patients with high PTEN expression had longer overall survival and disease-free survival.

Conclusion: miR-106b-5p promotes HCC stemness maintenance and metastasis by targeting PTEN via PI3K/Akt pathway. Inhibition of miR-106b-5p might be effective therapeutic strategies to treat advanced HCC.

Keywords: HCC, miRNAs, CSC-like properties, metastasis

\section{Introduction}

Hepatocellular carcinoma (HCC) is the fifth most aggressive cancer and has emerged as the third leading cause of cancer-related death globally. Only a small proportion of cases diagnosed at the early stage can be cured through surgical resection or liver transplantation. Most patients will develop into advanced disease owing to tumor recurrence or distant metastasis, and result in a dismal survival. ${ }^{1-3}$ Undoubtedly, tries made in the exploration of metastatic molecular targets and efficacious treatments for $\mathrm{HCC}$ are of great clinical significance.

Cancer stem cells (CSCs) are a small sub-population of cancer cells with self-renewal and tumor-initiating potential. ${ }^{4}$ They have been observed in numerous solid tumors, and exhibit vital roles in cancer heterogeneity, metastasis and drug resistance. ${ }^{5}$ With recent 
increase in CSC research, more and more investigators have verified that overexpression of several surface markers such as epithelial cell adhesion molecule (EpCAM), CD44 and CD133 in HCC cells could serve as an indicator of cancer stem-like properties. $^{6,7}$ The role of such cell populations in HCC invasion and metastasis, however, remains to be elucidated.

MicroRNAs (miRNAs) are a group of small noncoding single-stranded RNAs. They can act as tumor suppressors or promotors in tumorigenesis and metastasis via inhibition of target gene expression at post-transcriptional level in many human cancers. ${ }^{8}$ Cumulative evidence also suggests that miRNAs are effective modulators of CSC self-renewal and differentiation..$^{9-11}$ Here, we focused on miR-106b-5p, which was dysregulated in HCC tissues compared to corresponding cirrhotic tissues. ${ }^{12}$ Shi et al revealed that high serum level of miR-106b was positively associated with advanced stage of HCC patients. ${ }^{13}$ Others also showed an upregulated expression of miR-106b in HCC tissues and that miR-106b exerts an important function in the cell proliferation or apoptosis of HCC. ${ }^{14,15}$ However, the role of miR-106b in HCC CSC reprogramming remains unclear.

In this study, we verified that miR-106b-5p could promote HCC CSC maintenance and metastasis by targeting PTEN via the PI3K/AKT pathway and offer a probable strategy for the therapy of HCC metastasis.

\section{Methods HCC cell lines and animals}

The highly metastatic human HCC cell lines MHCC97L, MHCC97H and HCCLM3 were established at Liver Cancer Institute, Zhongshan Hospital, Fudan University, Shanghai, People's Republic of China. ${ }^{16,17}$ The low metastatic human HCC cell lines Bel-7402 and PLC/5 and a normal liver cell line Chang liver were obtained from the Shanghai Cell Bank, Chinese Academy of Sciences. All the cell lines were maintained in DMEM (Hyclone, Los Angeles, CA, USA), 10\% fetal bovine serum (Hyclone) and 1\% penicillin-streptomycin (Invitrogen, Waltham, MA, USA) in a humidified $5 \% \mathrm{CO}_{2}$ incubator at $37^{\circ} \mathrm{C}$. Male BALB/c nu/nu mice (6 weeks old; Shanghai Institute of Material Medicine, Chinese Academy of Science) were housed in specific pathogen-free conditions. All animal experiments were approved by the Animal Care Committee, Zhongshan Hospital, Shanghai, People's Republic of China. All animals received humane care according to the criteria outlined in the Guide for the Care and Use of Laboratory Animals prepared by the National Academy of Sciences and published by the National Institutes of Health.

\section{Patients and follow-up}

Twenty paired frozen primary HCC and adjacent normal liver tissues and another cohort of 81 paraffin-embedded HCC tissues were collected from Zhongshan Hospital (Fudan University, Shanghai, People's Republic of China), with the approval of the Zhongshan Hospital Research Ethics Committee. The approval number for clinical samples was B2017-065R. These patients underwent hepatectomies between June 2005 and June 2008, with no other anticancer treatment before surgery. Informed written consent was obtained from all patients. The fresh tissues were snap frozen in liquid nitrogen and stored at $-80^{\circ} \mathrm{C}$. Follow-up information was updated until October 2016.

\section{RNA isolation and qRT-PCR used for miRNA and mRNA assays}

Total RNAs were extracted with Trizol Reagent (Thermo Fisher Scientific, Waltham, MA, USA). For miRNA detection, first-strand DNA was synthesized from $2 \mu \mathrm{g}$ of total RNA, and real-time PCR was performed in triplicate by the SYBR Green PCR method using an All-in-One miRNA qPCR Detection kit (GeneCopoeia, Rockville, MD, USA). The primers for has-miR-106b-5p were purchased from GeneCopoeia. U6 was used as internal control. For mRNA detection, cDNA was prepared from $2 \mu \mathrm{g}$ of total RNA using a PrimeScript RT reagent kit (Takara Bio, Kyoto, Japan) and quantified in triplicate by using the SYBR Premix Ex Taq ${ }^{\mathrm{TM}}$ II (Takara Bio) with GAPDH as an internal control. The primers of PTEN and GAPDH were synthesized by Sangon Biotech (Shanghai, People's Republic of China) as follows: PTEN, 5'-TGGATTCGACTTAGACTTGACCT-3' (forward) and 5'-GGTGGGTTATGGTCTTCAAAAGG-3' (reverse); GAPDH, 5'-GGAGCGAGA TCCCTCCAAAAT-3' (forward) and 5'-GGCTGTTGTCATACTTCTCATGG-3' (reverse). The threshold cycle $(\mathrm{Ct})$ values were analyzed using the comparative $\mathrm{Ct}(-\Delta \mathrm{Ct})$ method. ${ }^{18}$ The level of targets was obtained by normalizing to the endogenous reference and relative to Chang liver.

\section{Oligonucleotides and siRNA transfection}

Oligonucleotides, including miR-106b-5p mimic, inhibitor and negative control, were designed and synthesized by Thermo Fisher Scientific. Small interfering RNAs (siRNAs) against PTEN and negative control were purchased from GeneChem (Shanghai, People's Republic of China). The constructs and oligonucleotides were transfected into HCC cells by using Lipofectamine 2000, according to the manufacturer's guidelines (Thermo Fisher Scientific). The final 
concentration was $100 \mathrm{~nm}$ for mimic or inhibitor and $100 \mathrm{~nm}$ for siRNA. Ubi-Luc-MCS-IRES-Puromycin-miR-106b-5p expression lentiviruses, mU6-MCS-Ubi-Luc-miR-106b-5p inhibitor lentiviruses and their corresponding negative controls were purchased from GeneChem. The miR-106b-5p expression lentiviruses were infected into PLC/5 and the inhibitor lentiviruses into HCCLM3. Stably expressed clones were selected by qRT-PCR.

\section{Migration and invasion assays}

Cell migration and invasion assays were performed using transwell chambers (Corning Incorporated, Corning, NY, USA). Treated cells were seeded in the upper compartment incubated in serum-free media, and the lower compartment was filled with complete medium supplemented with $10 \%$ FBS. After 24 hours, migratory and invasive cells on the bottom surface of the filters were fixed by $4 \%$ paraformaldehyde, and stained by $0.1 \%$ crystal violet solution (MedChem Express, Shanghai, People's Republic of China), and counted under an inverted microscope. The experiments were performed in triplicates.

\section{Western blot analysis}

Cell proteins were collected using a mixture of RIPA lysis buffer (Santa Cruz Biotechnology, Inc., Santa Cruz, CA, USA) and phenylmethanesulfonyl fluoride (Roche Ltd, Dublin, Ireland). About $30 \mu \mathrm{g}$ protein was subjected to $10 \%$ sodium dodecyl sulfate polyacrylamide gel electrophoresis (Sigma-Aldrich, St Louis, MO, USA), and the bands were transferred to polyvinylidene fluoride membranes (Hoffman-La Roche Ltd., Basel, Switzerland). The membranes were then blocked in 5\% nonfat milk and incubated with primary antibodies against PTEN, AKT, p-AKT, CD44, CD133, EpCAM (1:1,000; Abcam, Cambridge, UK), and subsequently with matched secondary antibodies (Cell Signaling Technology, Danvers, MA, USA). Signals for the expression of each protein were detected with the Bio-Rad Gel imaging system (Bio-Rad, Hercules, CA, USA). PI3K inhibitor LY294002 was purchased from LC Laboratories. GAPDH (G8140; US Biological, Swampscott, MA, USA) was used as a loading control.

\section{Luciferase reporter assay}

The binding sites for miR-106b-5p in the 3 -UTR sequence of PTEN were specifically synthesized. The mutant PTEN 3'-UTR was constructed by mutating multiple nucleotides complementary to the miR-106b-5p seed region. A mixture of $100 \mathrm{ng}$ pmiR-RB-Report ${ }^{\mathrm{TM}}$ hPTEN wild-type (WT) or mutant reporter plasmid vector together with $100 \mathrm{nM}$
miR-106b-5p mimics or negative control (Thermo Fisher Scientific) was co-transfected into HEK293T cells in 96-well plates using Lipofectamine 2000 (Thermo Fisher Scientific). Luciferase activities of the indicated cells were measured using the Dual Luciferase Reporter Assay System (Promega, Madison, WI, USA) 24 hours after transfection. Firefly and Renilla luciferase activities were measured using the Luc-Pair miRNA Luciferase Assay Kit (GeneCopoeia). Activities were normalized to that of Renilla luciferase.

\section{Tumor sphere formation assay}

Cells were cultured in DMEM-F12 (Sigma-Aldrich) supplemented with $20 \mathrm{ng} / \mathrm{mL}$ EGF (PeproTech, Rocky Hill, NJ, USA), $10 \mathrm{ng} / \mathrm{mL}$ FGF (PeproTech), $4 \mathrm{ng} / \mathrm{mL}$ insulin and B27 (Thermo Fisher Scientific) in Ultra-Low Attachment 6-well plates (Corning Incorporated). The cells were imaged under a microscope (Olympus Corporation, Tokyo, Japan) after 20 days of incubation.

\section{In vivo lung colonization assay}

Six-week-old male nude mice weighing 10-15 g were injected by tail vein using sterile 28 gauge needles and $300 \mu \mathrm{L}$ syringes (Hamilton Bonaduz A.G., Bonaduz, Switzerland) with $1 \times 10^{5}$ HCCLM3-miR-106b-5p-i, HCCLM3-NC, PLC/5-miR-106b-5p-o and PLC/5-NC cells, respectively. All mice were monitored once every 3 days and sacrificed 8 weeks later. Total metastatic foci were counted in paraffin-embedded lungs under a microscope, as described previously. ${ }^{19}$

\section{Immunohistochemical staining}

Immunohistochemistry for target molecules was performed on formalin-fixed paraffin tissues. The slides were probed with a primary antibody against PTEN (1:200; Abcam) and then incubated with horseradish peroxidase-conjugated IgG (1:500; Thermo Fisher Scientific), and the proteins were visualized in situ by using 3,3'-diaminobenzidine. The intensity of positive staining was measured with integrated optical density (IOD) as previously described. ${ }^{20}$

\section{Statistical analysis}

Data were analyzed using GraphPad Prism 6.0 software or Statistical Program for Social Sciences software 19.0 (SPSS, IBM, Chicago, IL, USA). Quantitative variables were expressed as means \pm SD and analyzed by Student's $t$-test, one-way ANOVA and Welch's $t$-tests. Qualitative variables were compared using Pearson $\chi^{2}$-test or Fisher exact test. Overall survival (OS) and disease-free survival (DFS) were 
estimated using Kaplan-Meier method, and the difference in survival was evaluated by log-rank tests. Results were considered statistically significant at $P<0.05$.

\section{Results}

\section{miR-106b-5p level is upregulated during HCC progression}

To determine the clinical significance of miR-106b-5p in HCC, The Cancer Genome Atlas (TCGA) (http://cancergenome.nih.gov/) database and GSE22058 were used to analyze miR-106b-5p expression in HCC tissues and adjacent non-cancerous liver tissues (ANLTs). A significantly higher level of miR-106b-5p was found in HCC tissues compared to ANLTs $(P<0.001$, Figure $1 \mathrm{~A}$ and $\mathrm{B})$. In addition, $75 \%$ $(16 / 20)$ of the HCC tissues from our liver institute showed an enhanced miR-106b-5p level compared to their corresponding ANLTs, when analyzed by qRT-PCR (Figure 1C).
To evaluate whether miR-106b-5p plays an essential role in HCC metastasis, we then examined the relationship between endogenous miR-106b-5p levels and metastatic potentials. In the normal liver cell line, Chang liver and five HCC cell lines (PLC/5, Bel-7402, MHCC97H, MHCC97L and HCCLM3, in ascending order of metastatic potential), the endogenous level of miR-106b-5p gradually increased (Figure 1D). Together, miR-106b-5p was abnormally upregulated as HCC progressed and could affect HCC metastasis.

\section{miR-106b-5p enhances HCC migration and invasion in vitro and in vivo}

Increased cell migration and invasion are pivotal steps for human cancer metastasis and recurrence. Therefore, we studied the effect of miR-106b-5p on HCC cell migration and invasion by loss- and gain-of-function experiments after transient transfections with $100 \mathrm{nM}$ miR-106b-5p mimics or
A

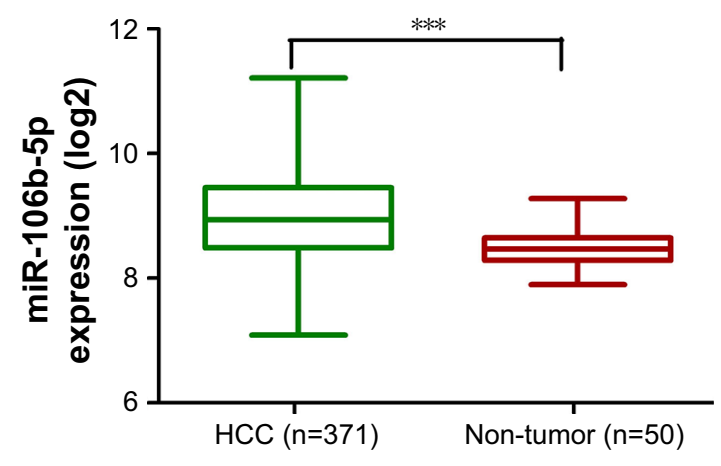

B

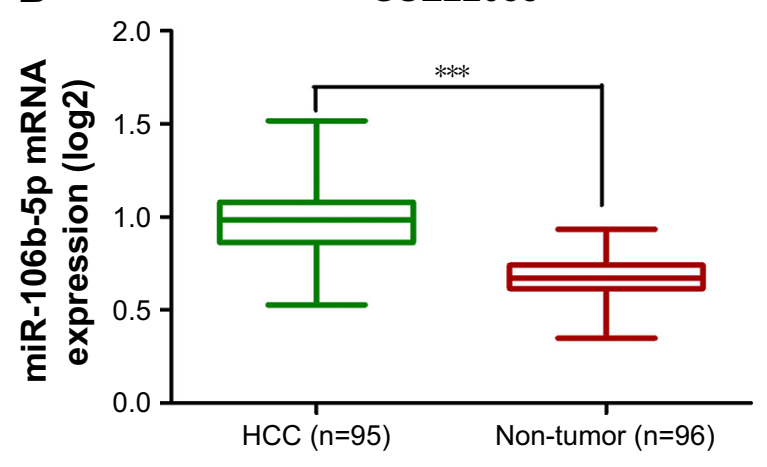

C

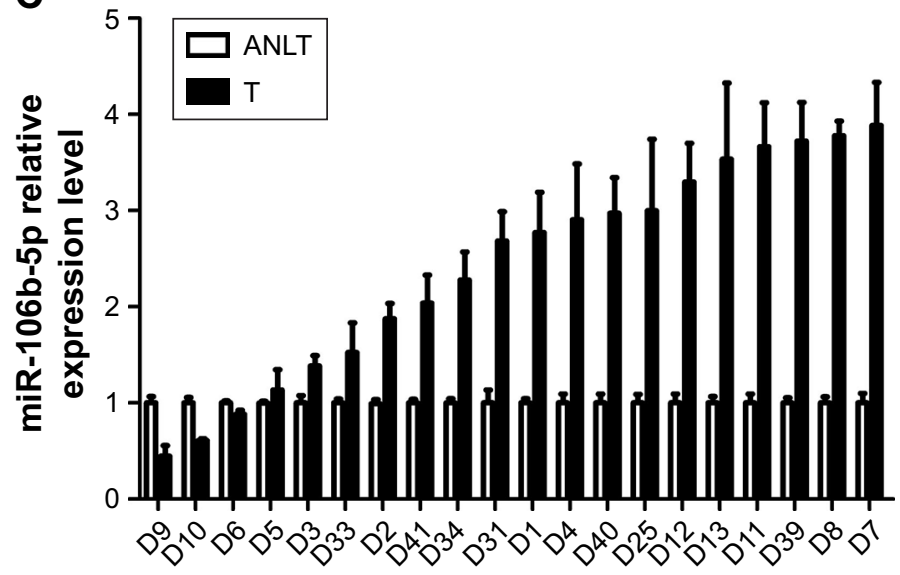

D

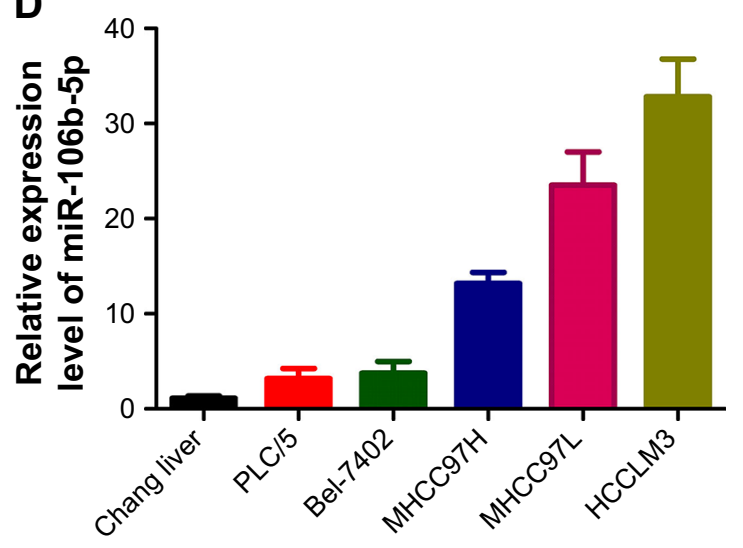

Figure I miR-106b-5p expression in HCC tissues, compared to ANLTs and a normal liver cell line.

Notes: miR-106b-5p expression level in human HCC tissues and ANLTs from TCGA (A) and GSE22058 database (B), ***P $<0.00$ I; qRT-PCR analysis to quantify the endogenous miR-106b-5p levels in Chang liver, PLC/5, Bel-7402, MHCC97H, MHCC97L and HCCLM3 cells (C) and 20 paired human HCC tissues and ANLTs (D). For cell lines, U6 was used as a control and data were normalized to the level of Chang liver cells.

Abbreviations: HCC, hepatocellular carcinoma; ANLT, adjacent non-tumor liver tissue; TCGA, The Cancer Genome Atlas; qRT-PCR, quantitative real-time polymerase chain reaction. 
inhibitors in vitro using high metastatic HCCLM3 and low metastatic PLC/5 cells. The miR-106b-5p inhibitor (miR$106 \mathrm{~b}-5 \mathrm{p}-\mathrm{i})$ significantly reduced the level of miR-106b-5p in HCCLM 3 cell lines by 0.6 -fold $(P<0.05$, Figure $2 \mathrm{~A})$ and miR-106b-5p mimics (miR-106b-5p-o) clearly upregulated the miR-106b-5p level in PLC/5 cell lines by 37.0-fold $(P<0.001$, Figure 2B). The migration and invasion capabilities of HCCLM3 cells were reduced by more than 0.3 - and 0.6 -fold, respectively, by miR-106b-5p-i, compared to WT and negative control (NC) cells $(P<0.05$, Figure 2C). By contrast, in $\mathrm{PLC} / 5$ cells, migration and invasion were increased by more than 0.6- and 0.4-fold with miR-106b-5p-o treatment, compared with their non-transfected counterparts $(P<0.001$, Figure 2D). These preliminary results indicated the positive regulatory roles of miR-106b-5p on in vitro HCC migration and invasion.

Colonization and outgrowth in a second organ is also an important part of the metastatic cascade. To check whether miR-106b-5p is involved in this step, a tail-vein injection model was successfully established in Balb/c nude mice after injection with miR-106b-5p stably overexpressed or knockdowned human HCC cells (Figure S1A and B). In 8 weeks, the pulmonary metastasis rate in HCCLM3-NC mice was $100 \%$ (6 of 6), which was significantly higher than that of
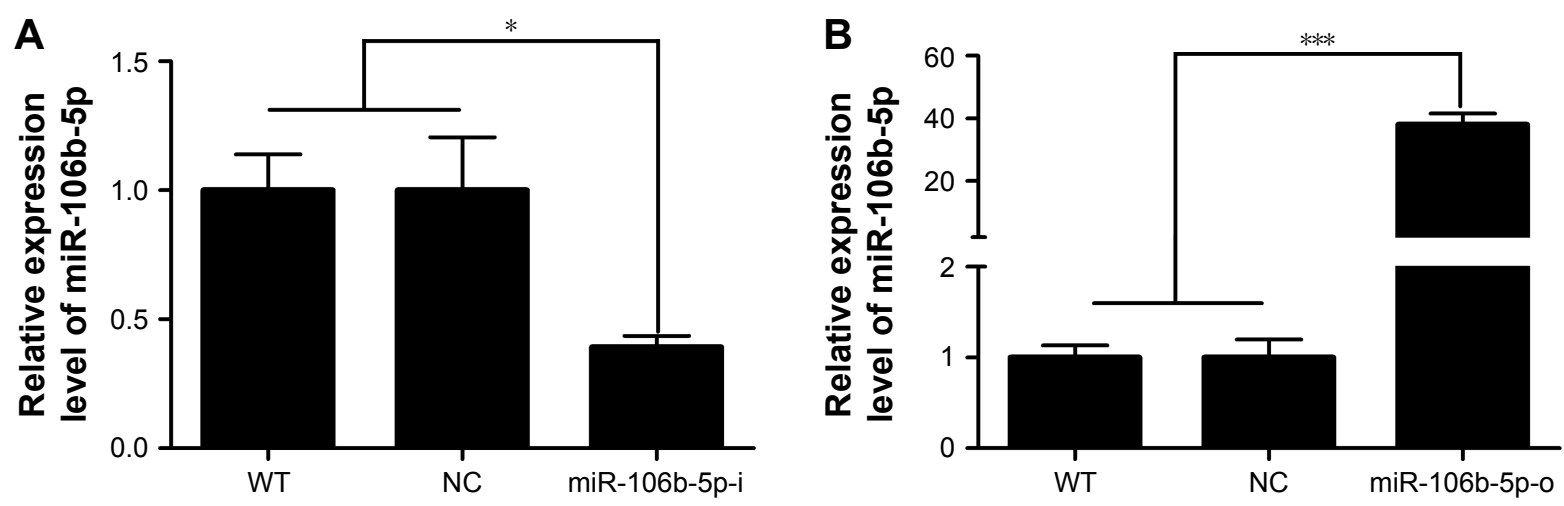

\section{C}

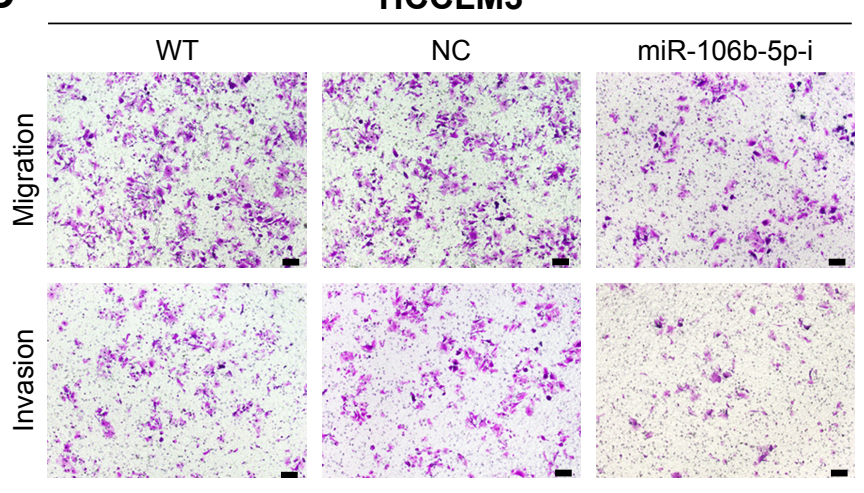

D

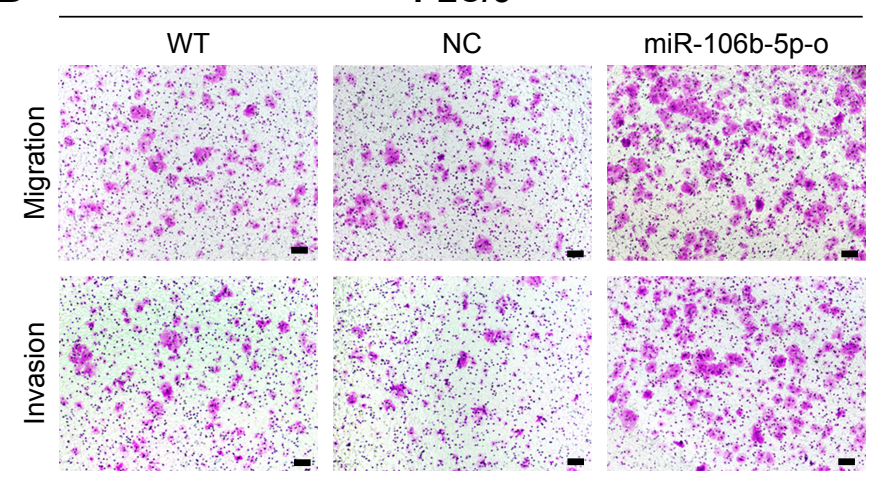

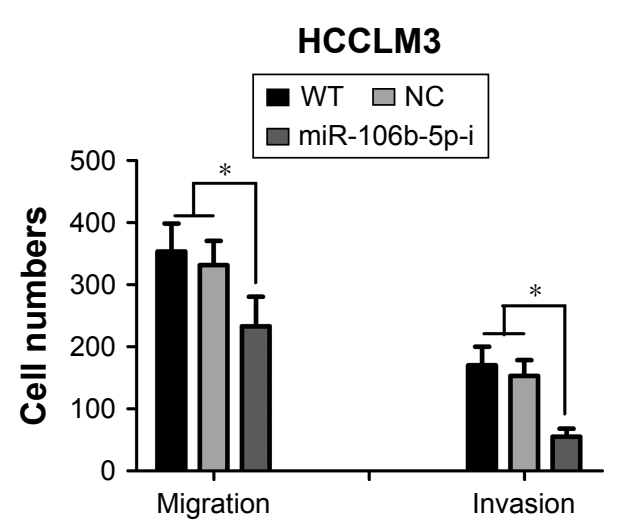

PLC/5

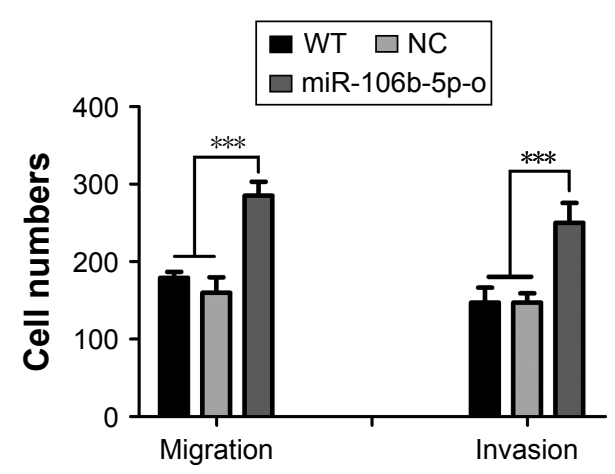

Figure 2 (Continued) 
E

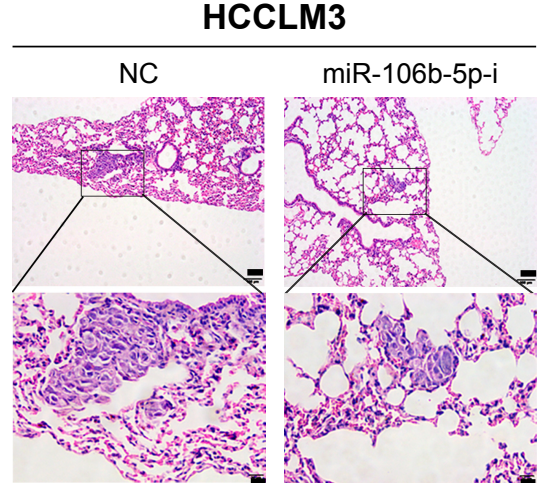

$\mathbf{F}$

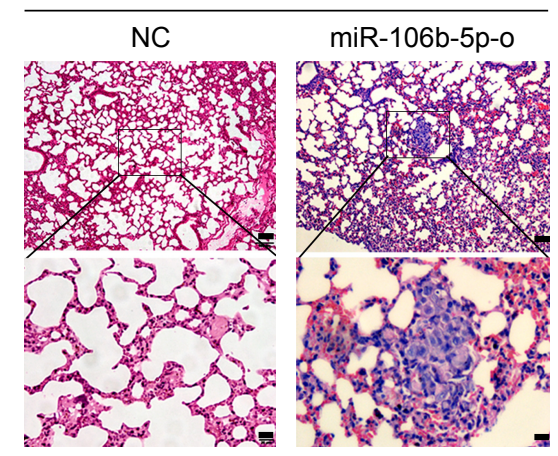

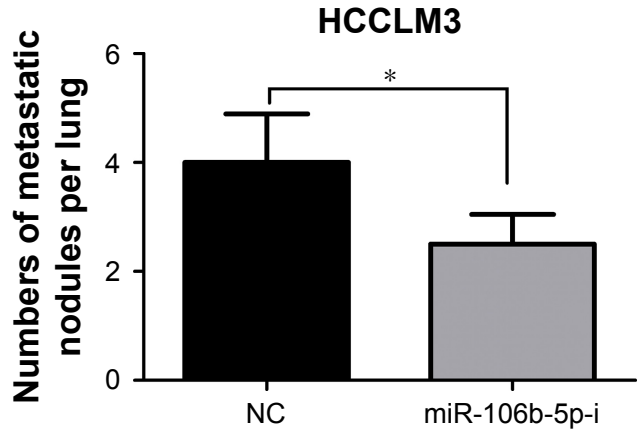

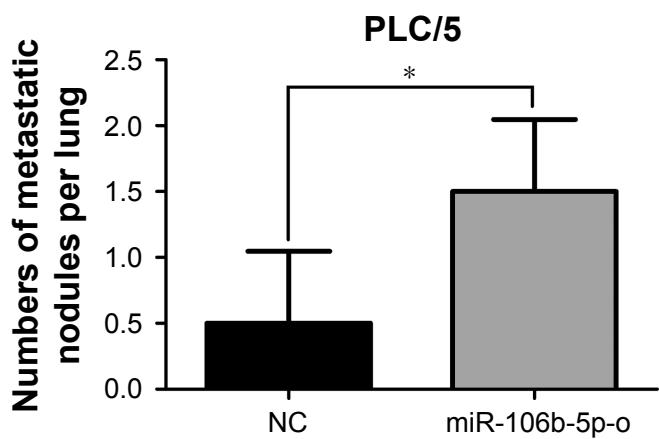

Figure 2 miR-106b-5p promotes human HCC metastasis in vitro and in vivo.

Notes: Evaluation of miR-106b-5p level in HCCLM3 cell transfected with miR-106b-5p inhibitors (miR-106b-5p-i) or NC (A) and PLC/5 cell transfected with miR-106b-5p mimics (miR-106b-5p-o) or NC (B) by qRT-PCR. Knockdown (C) or overexpression (D) of miR-I06b-5p inhibited or promoted HCC cell migration and invasion, respectively. Scale bars: $100 \mu \mathrm{m}$. H\&E staining (E) and number of lung colonization (F) in the recipient mice on day 60 after tail vein injection with $1.0 \times 10^{5} \mathrm{HCCLM3}$ and PLC/5 cells. Scale bars: (top) $100 \mu \mathrm{m}$, (bottom) $20 \mu \mathrm{m}$. $* P<0.05$, $* * * P<0.00$ I

Abbreviations: HCC, hepatocellular carcinoma; NC, negative control; qRT-PCR, quantitative real-time polymerase chain reaction; H\&E, hematoxylin and eosin; WT, wild-type.

HCCLM3-miR-106b-5p-i mice (50\%, 3 of 6) by histological examinations on lung metastatic foci. The numbers of metastatic nodules in HCCLM3-NC mice were also greater than those in HCCLM3-miR-106b-5p-i (Figure 2E). Meanwhile, 4 of 6 mice in PLC/5-miR-106b-5p-o group developed obvious pulmonary metastasis foci compared to only 2 mice in PLC/5-NC group. Overexpression of miR-106b-5p resulted in a 3.0-fold increase in PLC/5 tumor foci in the lungs of nude mice (Figure 2F). These observations suggested that ectopic forced expressions of miR-106b-5p conspicuously promoted HCC lung metastasis, and deficiencies of this miRNA suppressed tumor metastasis in vivo system.

\section{miR-I06b-5p promotes CSC-like properties of HCC}

In malignant cancer cells, acquisition of stem cell-like features is associated with enhanced migratory potential, such as tumor sphere formation and expression of stem cell surface markers. ${ }^{21}$ Therefore, we evaluated tumor sphere formation in miR-106b-5p mimics or inhibitors transfected HCC cells. The number of tumor spheres (with a diameter larger than $50 \mu \mathrm{m}$ ) was $5.5 \pm 0.8$ in miR-106b-5p-i HCCLM3 cells, significantly less than that in the WT and NC groups, which was $10 \pm 2.3,9.8 \pm 1.2$, respectively $(P<0.05$, Figure $3 \mathrm{~A})$. The number and size of tumor spheres in miR-106b-5p-o PLC/5 cells were remarkably greater than those in the WT and NC groups, which was 7.1 $\pm 0.9,3.0 \pm 1.0,2.8 \pm 0.6$, correspondingly $(P<0.01$; Figure $3 \mathrm{~B})$.

To confirm these results, three postulated biomarkers of CSCs (CD133, EpCAM and CD44) were studied in HCC cell lines pretreated with miR-106b-5p inhibitors or mimics. The protein levels of EpCAM, CD133 and CD44 were remarkably downregulated in HCCLM3-miR-106b-5p-i cells, and significantly upregulated in PLC/5-miR-106b-5p-o cells compared with the WT and NC groups (Figure 3C). These data suggest that miR-106b-5p plays a promoting role on HCC CSC-like properties.

\section{PTEN is a downstream target of miR-106b-5p}

To uncover the underlying mechanisms, bioinformatics programs (miRanda, TargetScan and PicTar) were used for 
A

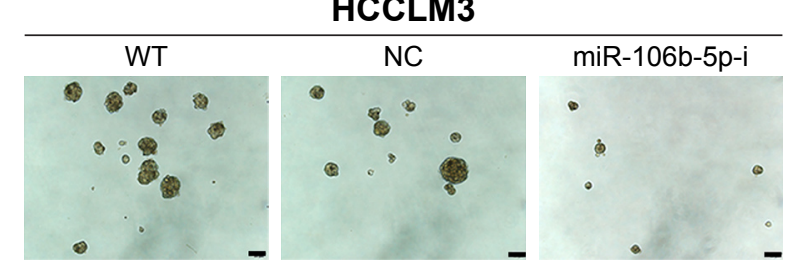

B

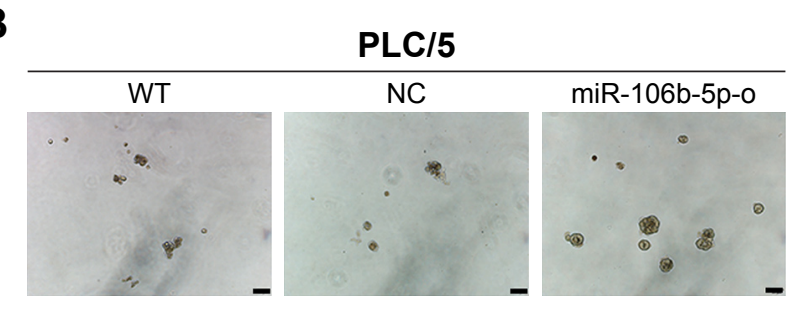

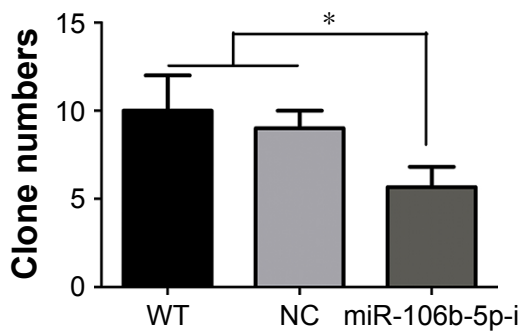

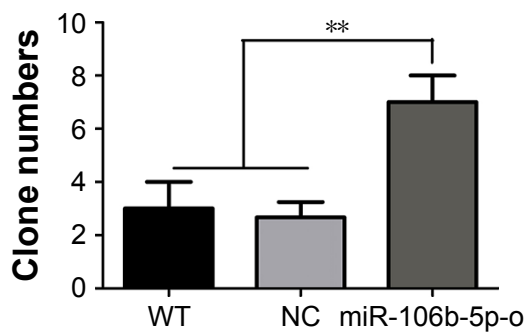

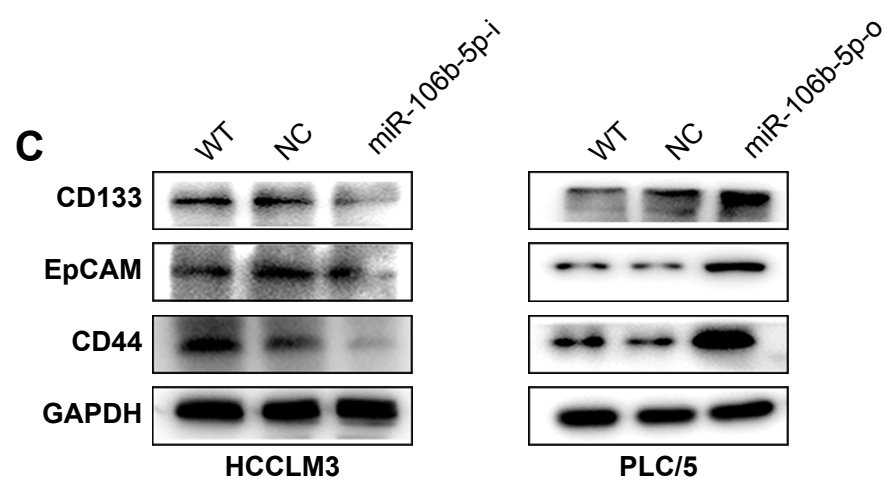

Figure 3 miR-106b-5p promotes CSC-like properties of HCC in vitro.

Notes: Representative images of HCCLM3 (A) and PLC/5 (B) tumor spheres $(>50 \mu \mathrm{m})$ and statistical results after indicated treatments. Scale bars: $50 \mu \mathrm{m}$. Western blot analysis of the CSC biomarkers CD44, CDI33 and EpCAM in HCCLM3 and PLC/5 cells after indicated treatments (C). $* P<0.05$, $* * P<0.01$.

Abbreviations: CSC, cancer stem cell; HCC, hepatocellular carcinoma; EpCAM, epithelial cell adhesion molecule; WT, wild type; NC, negative control.

miR-106b-5p targeted gene predictions. PTEN, a gene related to metastasis and stemness, ${ }^{22,23}$ was selected as a candidate (Figure 4A). PTEN protein levels were significantly increased in HCCLM3-miR-106b-5p-i cells and suppressed in PLC/5miR-106b-5p-o cells compared with WT and NC cells (Figure 4B). However, the phosphorylation level of AKT was negatively related with the expression level of PTEN in HCC cells (Figure 4B). In addition, PTEN was expressed at higher levels in the normal liver cell line and lower metastatic HCC cell lines, suggesting an inverse relationship between PTEN mRNA level and miR-106b-5p level (Figure 4C). Furthermore, luciferase reporter assay was performed to test the effect of miR-106b-5p on PTEN in HEK-293T cells expressing WT (2,251-2,271 nts) or mutant PTEN (Figure 4A). Twenty-four hours after co-transfection with miR-106b-5p mimics or scrambled (control), the luciferase activities were inhibited by $42 \%$ in WT transfected cells $(P<0.05$; Figure 4D). No significant changes were observed in mutant transfected cells. Thus, PTEN is a direct target of miR-106b-5p, and miR-106b-5p could induce the activity of PI3K/AKT signal pathway by targeting PTEN.

\section{Clinical characteristics of PTEN in HCC patients}

To investigate the clinical significance of PTEN in HCC patients, PTEN protein levels were evaluated in paratumor and cancerous tissues of HCC patients by immunohistochemistry and western blot analysis. As shown in Figure S2A and B, PTEN expression in cancerous tissues was significantly lower than that in the corresponding adjacent non-HCC tissues. Furthermore, there was an opposite relationship between PTEN expression and miR-106b-5p level in HCC tissues $(R=-0.627, P=0.003$; Figure S2C). Additionally, HCC tissues from our liver institute showed a decreased PTEN level compared to their corresponding ANLTs, when analyzed by western blot (Figure S2D). Also, downregulated 
A

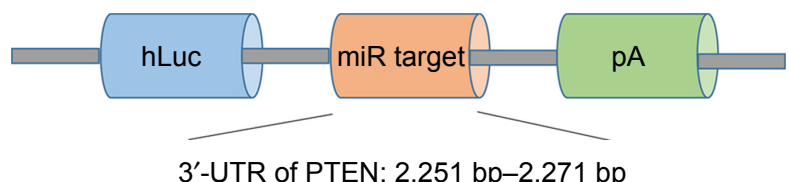

3'-UTR of PTEN: 2,251 bp-2,271 bp

Original sequence $\quad 5^{\prime}$ TTTTGCACTGTTAT TATTTT T 3

has-miR-106b-5p 3' UAGACGUGACAGUC IIIII I I

Mutant sequence $\quad 5^{\prime}$ TTTTGCACTGTTAT GCGGGG T 3'

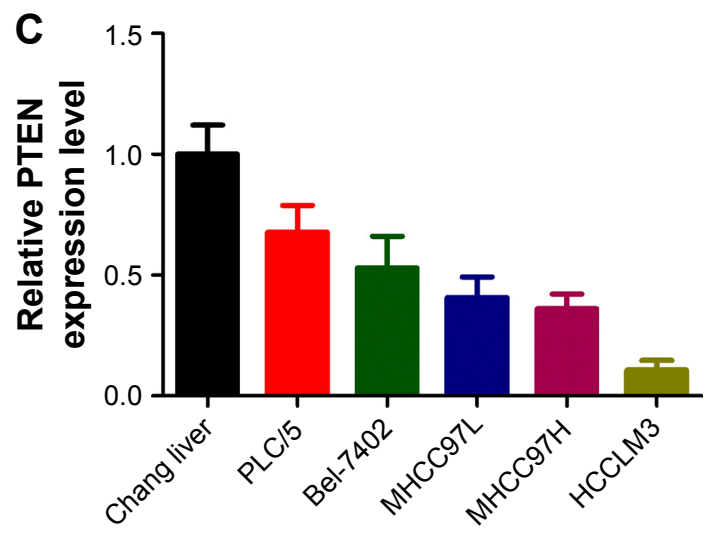

D

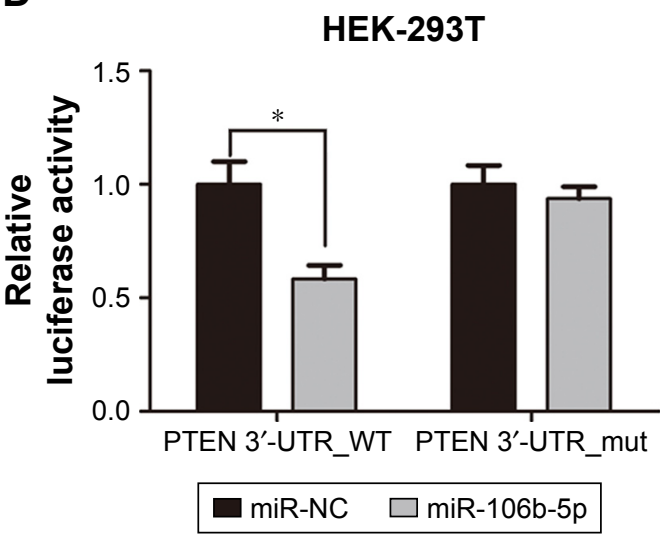

Figure 4 Direct regulation of PTEN expression by miR-106b-5p.

Notes: Schematic diagram of the dual luciferase miRNA target reporter vector $(\mathbf{A})$, the wild type and mutant PTEN 3'-UTR sequences are shown with the miR-I06b-5P sequence. (B) Western blot analysis of PTEN protein level in HCCLM3 cells transfected with miR-106b-5p inhibitors or NC (left), and PLC/5 cells transfected with miR-106b-5p mimics or NC (right). (C) qRT-PCR analysis of PTEN mRNA levels in five HCC cancer cell lines with different metastatic potentials and a normal liver cell line. (D) Luciferase assays in HEK293T cells with wild-type or mutant PTEN 3'-UTR vectors and miR-106b-5P mimic or NC. $* P<0.05$.

Abbreviations: PTEN, phosphatase and tensin homolog; HCC, hepatocellular carcinoma; WT, wild type; NC, negative control; qRT-PCR, quantitative real-time polymerase chain reaction.

mRNA level of PTEN was observed in 371 HCC tissues compared with 50 adjacent non-cancerous liver tissues by analyzing TCGA database ( $P<0.001$; Figure S2E).

Then, a cohort of $81 \mathrm{HCC}$ tissues was enrolled for further analysis and was dichotomized into low- and highexpression groups based on IOD. Our results showed that PTEN was inversely correlated with tumor size $(P=0.003)$, microvascular invasion $(P=0.032)$ and pathological stage $(P=0.038)$ (Table S1). Kaplan-Meier analysis showed that patients with high PTEN expression had markedly longer OS (log-rank, $P=0.01)$ and DFS (log-rank, $P=0.018$ ) than those with low PTEN expression, and the 5-year OS and DFS rate were prolonged from 0.34 to 0.45 and from 0.21 to 0.30 , respectively (Figure S2F). Taken together, PTEN showed a favorable correlation with HCC prognosis, suggesting that it could be used as a good prognostic biomarker for HCC. Also, PTEN was the direct downstream target for miR-106b-5p in HCC.

\section{Activation or inhibition of PTEN/PI3K/ AKT pathway mimicked or abrogated the effects of miR-106b-5p on HCC metastasis and stemness}

To further elucidate the importance of PTEN in tumor cell metastasis and stemness, siRNA was employed to downregulate the expression of PTEN in HCCLM3. As shown in Figure 5A, the effects of miR-106b-5p-i on the expression of PTEN and p-AKT were reversed by inhibition of PTEN in HCCLM3. Additionally, the levels of CD44, CD133 and EpCAM in HCCLM3 cells were dramatically reduced by miR-106b-5p-i, which were restored by si-PTEN (Figure 5B). Knockdown of PTEN promoted the formation of tumor spheres $(18 \pm 3,30 \pm 2$, $5 \pm 2$ vs $16 \pm 2, P<0.001)$, migration $(85 \pm 11,165 \pm 13,22 \pm 4$ vs $47 \pm 6, P<0.001)$ and invasion $(66 \pm 11,145 \pm 14,19 \pm 4$ vs $46 \pm 8, P<0.001$ ) of HCCLM3 cells (Figure $5 \mathrm{C}$ and $\mathrm{D}$ ). Next, 2-(4-morpholinyl)-8-phenyl-chromone (LY294002), 
A

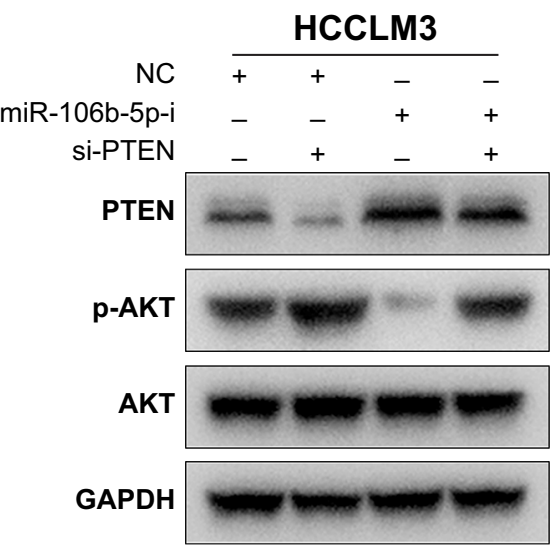

B

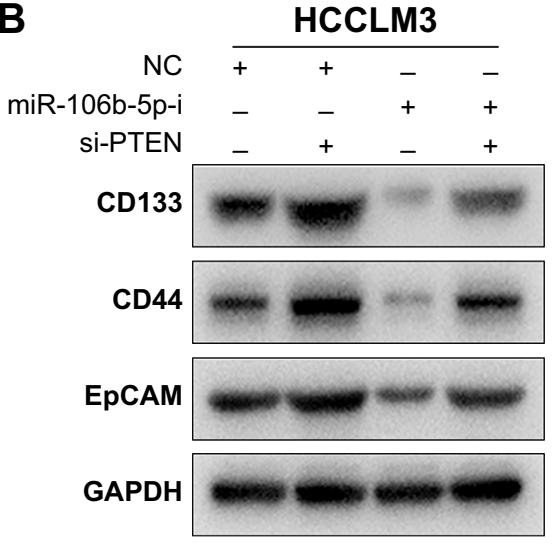

C

NC

miR-106b-5p-i Si-PTEN
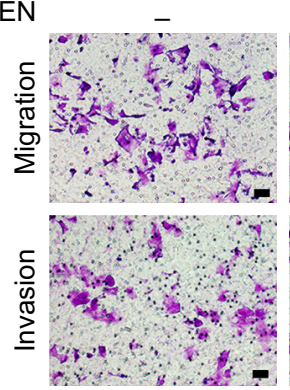

HCCLM3

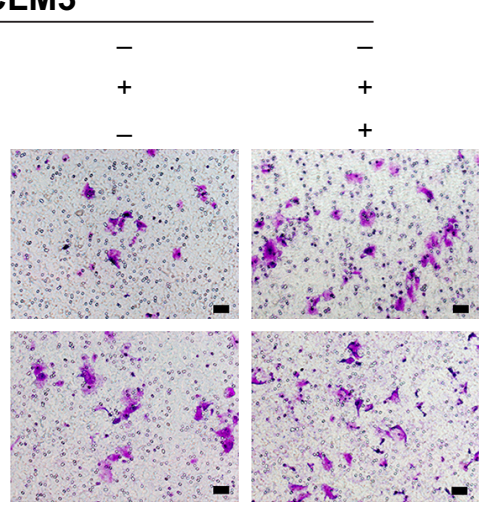

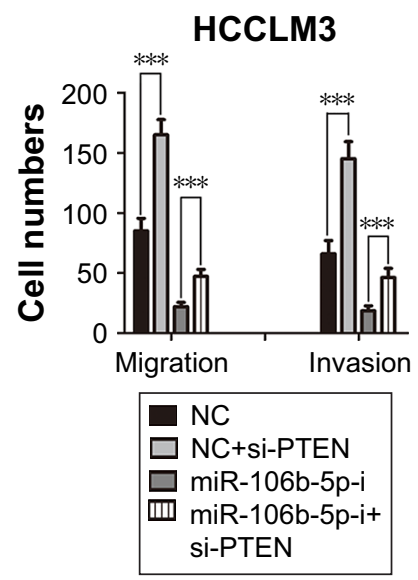

HCCLM3

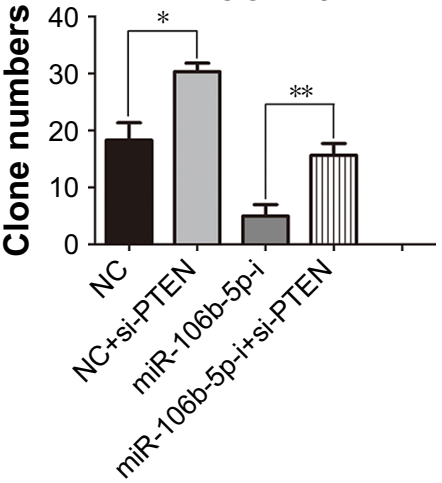

Figure 5 miR-106b-5p enhanced CSC characters and invasion capabilities by targeting PTEN.

Notes: Western blotting showed that the increased PTEN expression through miR-106b-5p inhibitor treatment was suppressed by si-PTEN followed with the reactivation of PI3K/AKT pathway $(\mathbf{A})$, and the inhibitory effect of miR-106b-5p-i on the stemness of HCCLM3 was reversed by si-PTEN (B). Migration and invasion capacities of HCCLM3 cells transfected with miR-106b-5p inhibitor were restored by PTEN knockdown. Representative images of invading cells on the membrane and statistical results from at least three independent experiments (C). Scale bars: $100 \mu \mathrm{m}$. (D) The stemness of HCCLM3 cells transfected with miR-106b-5p inhibitor was restored by PTEN knockdown. Representative images of tumor sphere numbers and statistical results from at least three independent experiments. Scale bars: $100 \mu \mathrm{m}$. $* P<0.05$, $* * P<0.0 \mathrm{I}$, $* * * P<0.00 \mathrm{I}$. Abbreviations: CSC, cancer stem cell; PTEN, phosphatase and tensin homolog; NC, negative control.

a specific PI3K inhibitor, was used to incubate PLC/5 cells for 48 hours. Western blotting demonstrated that the high expression of p-AKT induced by miR-106b-5p overexpression was downregulated by LY294002 (Figure 6A). The CSC properties of PLC/5 cells were attenuated, as indicated by the changes of protein levels of CD44, CD133 and EpCAM (Figure 6B). Moreover, transwell assays and tumor sphere formation assays showed that LY294002 abolished the effects of miR-106b-5p on the migration $(64 \pm 11,27 \pm 7,176 \pm 7$ vs $76 \pm 15 ; P<0.001)$, invasion $(65 \pm 6,24 \pm 5,165 \pm 8$ vs $81 \pm 3, P<0.001)$ and stemness $(11 \pm 4,3 \pm 1,42 \pm 3$ vs $18 \pm 3 ; P<0.001)$ of $P L C / 5$ cells (Figure 6C and D). All these results once again supported that PTEN was the functional target of miR-106b-5p- and 
A

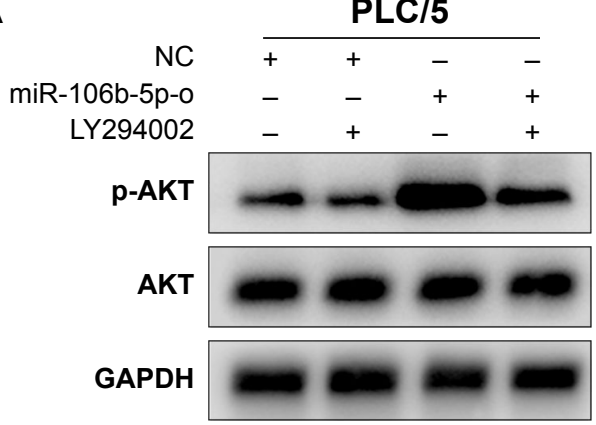

B

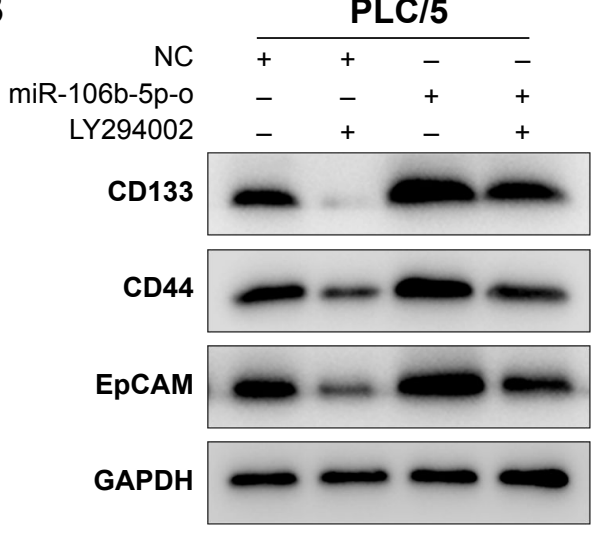

C
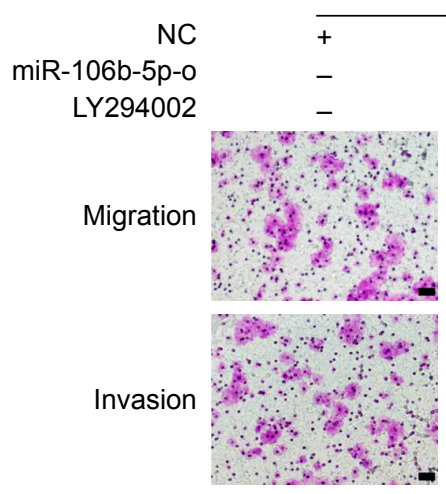

\section{PLC/5}

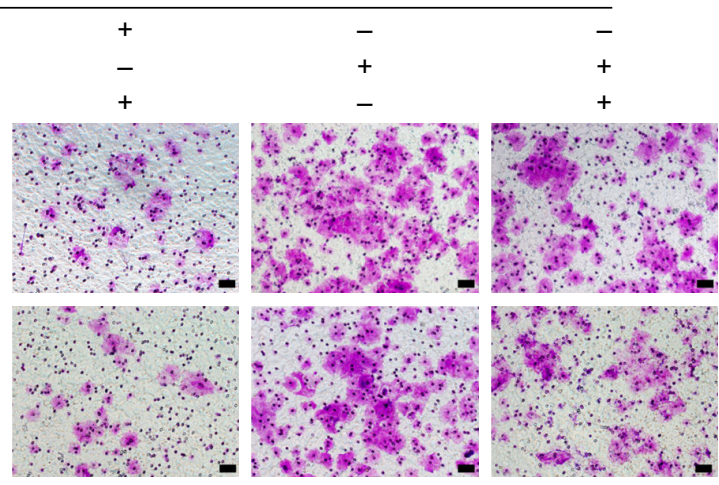

PLC/5

D

NC

miR-106b-5p-o LY294002

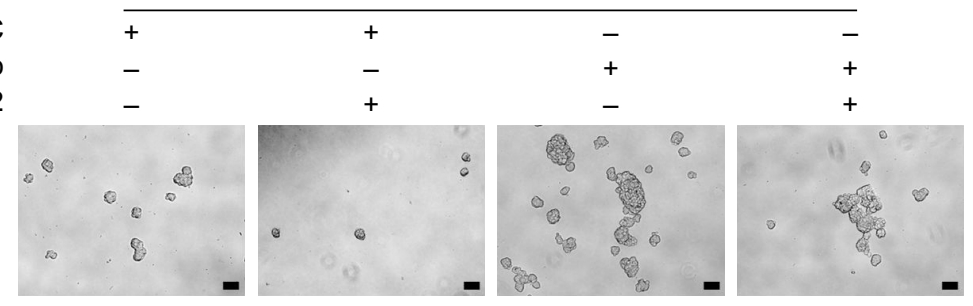

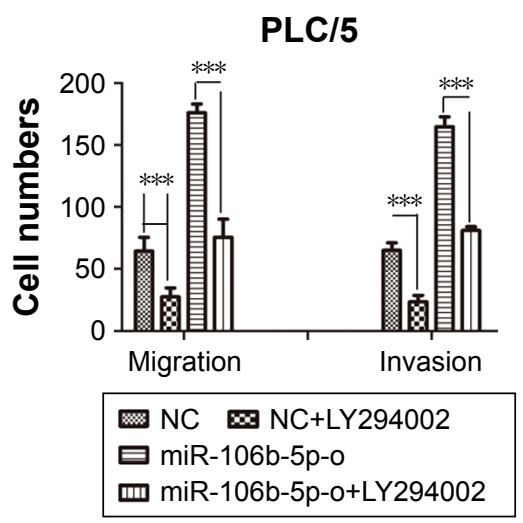

PLC/5

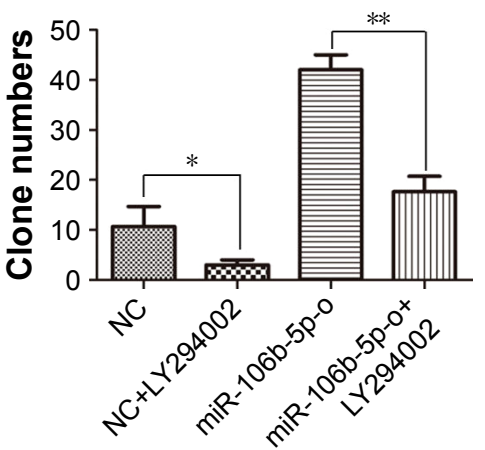

Figure 6 The enhanced CSC characters and metastatic abilities by miR-106b-5p overexpression could be abolished via the suppression of PTEN/PI3K/AKT pathway. Notes: Western blotting showed that P-AKT level was downregulated in PLC/5 cells after LY294002 treatment (A), followed by the protein changes of CSC markers in PLC/5 cells (B). Transwell assays were performed to evaluate the effects of LY294002 on migration and invasion of PLC/5 cells with overexpression of miR-106b-5p or NC (C). Scale bars: $100 \mu \mathrm{m}$. Image showing the capability changes of sphere forming of PLC/5 cells after LY294002 treatment (D). Scale bars: $100 \mu \mathrm{m}$. $* P<0.05$, $* * P<0.01$, $* * * P<0.001$.

Abbreviations: CSC, cancer stem cell; PTEN, phosphatase and tensin homolog; NC, negative control.

miR-106b-5p-mediated metastasis and CSC characters via PI3K/AKT signaling by targeting PTEN in HCC cells.

\section{Discussion}

HCC, which accounts for $90 \%$ of primary liver cancers, is the third leading cause of cancer-related mortality in the world. ${ }^{2}$ Most HCC cases underwent a chronic liver disease and had different degrees of cirrhosis. ${ }^{24}$ The most common risk factors for $\mathrm{HCC}$ are hepatitis B virus (HBV) and hepatitis C virus $(\mathrm{HCV})$ infection, with a worldwide incidence of $54 \%$ and $31 \%$, respectively. Other risk factors include alcohol abuse, obesity, hemochromatosis psychosis, environmental pollutants and so on. ${ }^{25}$ Taken into account the diversity of etiologies, HCC is, accordingly, an intricate disease characterized by gradual accumulation of genomic and epigenetic alterations, such as mutations, amplifications or deletions, 
translocations, dysregulation of non-coding RNAs and so on. ${ }^{26}$ miRNAs, as one kind of non-coding RNAs, play crucial roles in $\mathrm{HBV}, \mathrm{HCV}$ and non-alcoholic fatty liver diseaseinduced HCC development and could be used as potential non-invasive biomarkers for diagnosis especially when a definitive relationship between specific miRNAs profiles in serum/plasma of HCC is defined. For example, a panel of miRNAs (miR-21, miR-122, miR-125a/b, miR199a/b, miR-221, miR-222, miR-223, miR-224) has been identified as effective molecular markers for the assessment of early diagnosis and prognosis in $\mathrm{HBV}$ - or $\mathrm{HCV}$-positive $\mathrm{HCC}$ patients. ${ }^{27}$ Mounting evidences have emerged that miRNAs are the major drivers on tumor development. ${ }^{28,29}$ Therefore, identifications of metastasis-related miRNAs and their direct target genes are critical steps for understanding miRNA mechanisms on HCC metastatic progression. ${ }^{30-32}$ In this study, we focused on miR-106b-5p, which is significantly increased in $\mathrm{HCC}$ tissues relative to corresponding cirrhotic tissues. ${ }^{12}$ Quantitative analysis of our own samples and data from the TCGA and GSE22058 database showed elevated levels of miR-106b-5p in HCC tissues compared to paired non-tumor liver tissues. In addition, higher miR-106b-5p levels were observed in $\mathrm{HCC}$ cell lines than in normal liver cell lines. Furthermore, HCC cell lines with high metastatic potentials (MHCC97H, MHCC97L and HCCLM3) exhibited higher miR-106b-5p levels than did HCC cell lines with low metastatic potencies (PLC/5 and Bel-7402), suggesting a positive association of miR-106b-5p and aggressive HCC phenotype. We then explored the biological function of this miRNA in HCC metastasis. In vitro loss- or gain-of-function studies demonstrated that miR-106b-5p promoted HCC invasion and metastasis. Further, miR-106b-5p enhanced CSC-like properties of $\mathrm{HCCs}$, with increased tumor sphere formation and the upregulation of common CSC biomarkers (EpCAM, CD44 and CD133), suggesting that this miRNA is an effective regulator of CSC division, differentiation and tumor metastasis.

Since miR-106b-5p has a number of predicted targets in distinct cell types, we selected PTEN for further study owing to its critical correlation with stemness maintenance and tumor metastasis. In the current study, the mRNA level and protein expression of PTEN in HCC tissues (TCGA and our samples) and cells were significantly lower than that in nontumor liver tissues and cells. Besides, a negative relationship between miR-106b-5p and PTEN was also observed in the HCC cell lines and tissues. Luciferase reporter assay further showed that PTEN is a direct target of miR-106b-5p. PTEN, known as phosphatase and tensin homolog, is a classical tumor suppressor. Loss-function of PTEN mutation or deletion has been identified in various types of cancers. It facilitates the conversion of phosphatidylinositol-3,4,5-trisphosphate into the diphosphate form. ${ }^{33}$ Thus, PTEN could antagonize PI3K signaling and inhibit tumor progression. Recent researches have brought insight into the connection between CSCs property and PTEN/PI3K/AKT pathways. ${ }^{23}$ To evaluate the clinical signature of PTEN in HCC, 81 HCC patients from our liver cancer institute were artificially divided into high- and low-PTEN-expressing groups with IOD. Low expression of PTEN was correlated with malignant characteristics, including larger tumor size, increased microvascular invasion and more advanced pathological stage. High levels of PTEN in tumor tissues predicted good prognostic values in terms of OS and DFS in patients with HCC. Silencing of PTEN or AKT signaling offsets the suppressive role of miR-106b-5p inhibitor in metastatic abilities and cancer stem property of HCCLM3 cell lines, suggesting that PTEN is a functional target of miR-106b-5p. The data supported that miR-106b-5p could function as a pivotal regulator of HCC metastasis and CSClike properties by targeting PTEN via PI3K/AKT signaling.

Since the induction of CSC-like properties has been critically discussed as the key feature in tumor aggressiveness and metastasis, ${ }^{34-36}$ our findings in the present study demonstrate that miR-106b-5p as an oncogenic miRNA could promote tumor metastasis and invasion partly by stimulating CSClike properties through repression of PTEN. The data also suggest that miR-106b-5p could be an effective biomarker and potential therapeutic target in HCC patients in the future. However, whether other signal pathways that may modulate CSC-like maintenance and could be mediated by miR$106 b-5 p$ were not investigated in this study. Moreover, the effect of the HCC environment on miR-106b-5p expression or CSC-like properties was not explored.

\section{Conclusion}

miR-106b-5p promotes HCC stemness maintenance and metastasis by targeting PTEN via PI3K/Akt pathway. Inhibition of miR-106b-5p might be an effective therapeutic strategy to treat advanced HCC.

\section{Acknowledgments}

The work was jointly supported by the National Science Foundation of China (81272437 and 81472675) and the National Key Research and Development Plan (2016YFC0902400). We sincerely thank Professor Jian Zhou and Jia Fan from Liver Cancer Institute, Zhongshan Hospital, Shanghai, People's Republic of China, for their advice on study design. 


\section{Disclosure}

The authors report no conflicts of interest in this work.

\section{References}

1. Lau WY, Lai EC. Hepatocellular carcinoma: current management and recent advances. Hepatobiliary Pancreat Dis Int. 2008;7(3): 237-257.

2. Desai JR, Ochoa S, Prins PA, He AR. Systemic therapy for advanced hepatocellular carcinoma: an update. J Gastrointest Oncol. 2017;8(2): 243-255.

3. Finn RS, Zhu AX, Farah W, et al. Therapies for advanced stage hepatocellular carcinoma with macrovascular invasion or metastatic disease: a systematic review and meta-analysis. Hepatology. Epub 2017 Sep 7.

4. Liu BB, Qin LX, Liu YK. Adult stem cells and cancer stem cells: tie in or tear apart? J Cancer Res Clin Oncol. 2005;131(10):631-638.

5. DeSano JT, Xu L. MicroRNA regulation of cancer stem cells and therapeutic implications. AAPS J. 2009;11(4):682-692.

6. Ma S, Chan KW, Hu L, et al. Identification and characterization of tumorigenic liver cancer stem/progenitor cells. Gastroenterology. 2007; 132(7):2542-2556.

7. Yamashita T, Ji J, Budhu A, et al. EpCAM-positive hepatocellular carcinoma cells are tumor-initiating cells with stem/progenitor cell features. Gastroenterology. 2009;136(3):1012-1024.

8. Bartel DP. MicroRNAs: genomics, biogenesis, mechanism, and function. Cell. 2004;116(2):281-297.

9. Zimmerman AL, Wu S. MicroRNAs, cancer and cancer stem cells. Cancer Lett. 2011;300(1):10-19.

10. Yang ZF, Ho DW, Ng MN, et al. Significance of CD90+ cancer stem cells in human liver cancer. Cancer Cell. 2008;13(2):153-166.

11. Hatfield SD, Shcherbata HR, Fischer KA, Nakahara K, Carthew RW, Ruohola-Baker H. Stem cell division is regulated by the microRNA pathway. Nature. 2005;435(7044):974-978.

12. Pineau P, Volinia S, McJunkin K, et al. miR-221 overexpression contributes to liver tumorigenesis. Proc Natl Acad Sci U S A. 2010; 107(1):264-269.

13. Shi BM, Lu W, Ji K, Wang YF, Xiao S, Wang XY. Study on the value of serum miR-106b for the early diagnosis of hepatocellular carcinoma. World J Gastroenterol. 2017;23(20):3713-3720.

14. Shen G, Jia H, Tai Q, Li Y, Chen D. miR-106b downregulates adenomatous polyposis coli and promotes cell proliferation in human hepatocellular carcinoma. Carcinogenesis. 2013;34(1):211-219.

15. Xu C, Shi L, Chen W, et al. MiR-106b inhibitors sensitize TRAILinduced apoptosis in hepatocellular carcinoma through increase of death receptor 4. Oncotarget. 2017;8(26):41921-41931.

16. Li Y, Tang Y, Ye L, et al. Establishment of a hepatocellular carcinoma cell line with unique metastatic characteristics through in vivo selection and screening for metastasis-related genes through cDNA microarray. $J$ Cancer Res Clin Oncol. 2003;129(1):43-51.

17. Li Y, Tang ZY, Ye SL, et al. Establishment of cell clones with different metastatic potential from the metastatic hepatocellular carcinoma cell line MHCC97. World J Gastroenterol. 2001;7(5):630-636.

18. Landgraf P, Rusu M, Sheridan R, et al. A mammalian microRNA expression atlas based on small RNA library sequencing. Cell. 2007; 129(7):1401-1414.
19. Ye QH, Qin LX, Forgues M, et al. Predicting hepatitis B virus-positive metastatic hepatocellular carcinomas using gene expression profiling and supervised machine learning. Nat Med. 2003;9(4):416-423.

20. Zhu XD, Zhang JB, Zhuang PY, et al. High expression of macrophage colony-stimulating factor in peritumoral liver tissue is associated with poor survival after curative resection of hepatocellular carcinoma. J Clin Oncol. 2008;26(16):2707-2716.

21. Zhang W, Mu D, Feng K. Hierarchical potential differentiation of liver cancer stem cells. Adv Clin Exp Med: official organ Wroclaw Medical University. 2017;26(7):1137-1141.

22. Dubrovska A, Kim S, Salamone RJ, et al. The role of PTEN/Akt/PI3K signaling in the maintenance and viability of prostate cancer stem-like cell populations. Proc Natl Acad Sci U S A. 2009;106(1):268-273.

23. Ciuffreda L, Falcone I, Incani UC, et al. PTEN expression and function in adult cancer stem cells and prospects for therapeutic targeting. Adv Biol Regul. 2014;56:66-80.

24. Xu J, Li J, Zheng TH, Bai L, Liu ZJ. MicroRNAs in the occurrence and development of primary hepatocellular carcinoma. Adv Clin Exp Med. 2016;25(5):971-975.

25. Mizuguchi Y, Takizawa T, Yoshida H, Uchida E. Dysregulated miRNA in progression of hepatocellular carcinoma: a systematic review. Hepatol Res. 2016;46(5):391-406.

26. Wahid B, Ali A, Rafique S, Idrees M. New insights into the epigenetics of hepatocellular carcinoma. BioMed Res Int. 2017;2017:1609575.

27. Fiorino S, Bacchi-Reggiani ML, Visani M, et al. MicroRNAs as possible biomarkers for diagnosis and prognosis of hepatitis B- and C-related-hepatocellular-carcinoma. World J Gastroenterol. 2016; 22(15):3907-3936.

28. Tan Y, Ge G, Pan T, Wen D, Gan J. A pilot study of serum microRNAs panel as potential biomarkers for diagnosis of nonalcoholic fatty liver disease. PLoS One. 2014;9(8):e105192.

29. Schubert M, Junker K, Heinzelmann J. Prognostic and predictive miRNA biomarkers in bladder, kidney and prostate cancer: Where do we stand in biomarker development? J Cancer Res Clin Oncol. 2016; 142(8):1673-1695.

30. Yang X, Zhang XF, Lu X, et al. MicroRNA-26a suppresses angiogenesis in human hepatocellular carcinoma by targeting hepatocyte growth factor-cMet pathway. Hepatology. 2014;59(5):1874-1885.

31. Chang RM, Yang H, Fang F, Xu JF, Yang LY. MicroRNA-331-3p promotes proliferation and metastasis of hepatocellular carcinoma by targeting $\mathrm{PH}$ domain and leucine-rich repeat protein phosphatase. Hepatology. 2014;60(4):1251-1263.

32. Li S, Yang $\mathrm{C}$, Zhai L, et al. Deep sequencing reveals small RNA characterization of invasive micropapillary carcinomas of the breast. Breast Cancer Res Treat. 2012;136(1):77-87.

33. Das S, Dixon JE, Cho W. Membrane-binding and activation mechanism of PTEN. Proc Natl Acad Sci U S A. 2003;100(13):7491-7496.

34. Doherty MR, Smigiel JM, Junk DJ, Jackson MW. Cancer stem cell plasticity drives therapeutic resistance. Cancers (Basel). 2016;8(1):E8.

35. Flores-Téllez TN, Villa-Treviño S, Piña-Vázquez C. Road to stemness in hepatocellular carcinoma. World J Gastroenterol. 2017;23(37): 6750-6776.

36. Deshmukh A, Binju M, Arfuso F, Newsholme P, Dharmarajan A. Role of epigenetic modulation in cancer stem cell fate. Int J Biochem Cell Biol. 2017;90:9-16. 


\section{Supplementary materials}

A

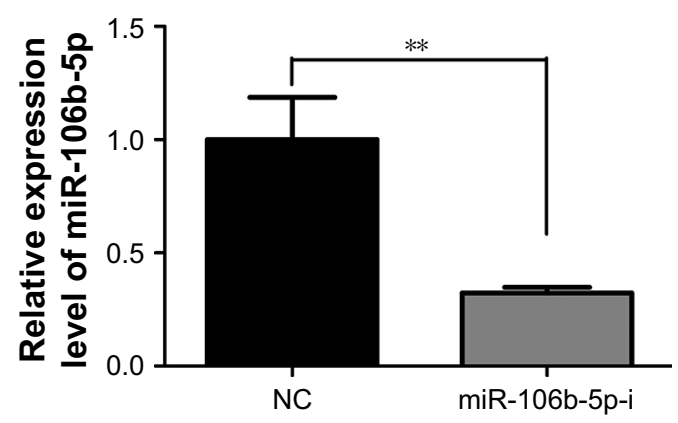

B

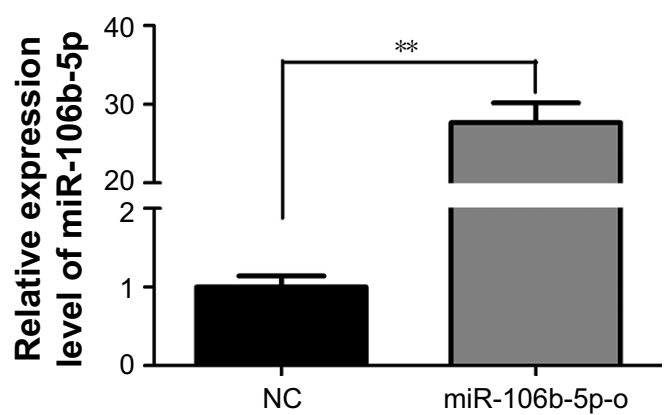

Figure SI The miR-106b-5p level in HCC cell lines stably transfected with miR-106b-5p overexpression or knockdown of lentiviral vector virus.

Notes: HCCLM3 cells' stable transduction with NC or miR-106b-5p knockdown virus (miR-106b-5p-i) was confirmed by qRT-PCR (A). PLC/5 cells' stable transduction with NC or miR-106b-5p overexpression virus (miR-106b-5p-o) was confirmed by qRT-PCR (B). $* * P<0.01$.

Abbreviations: HCC, hepatocellular carcinoma; qRT-PCR, quantitative real-time polymerase chain reaction; NC, negative control.

A

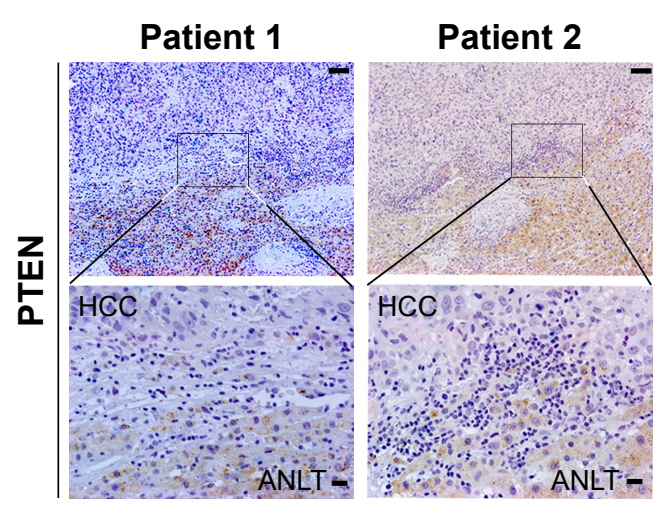

C

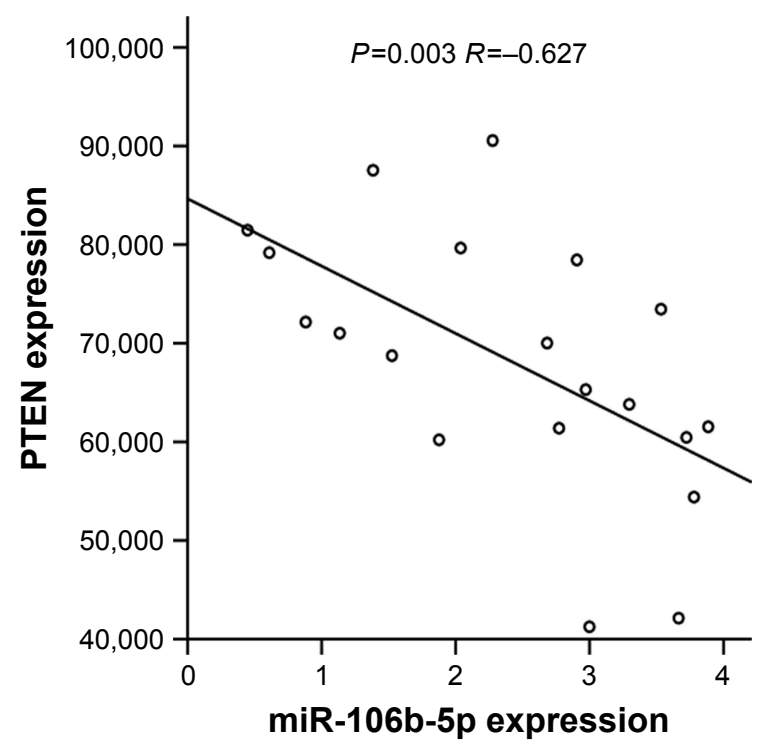

B

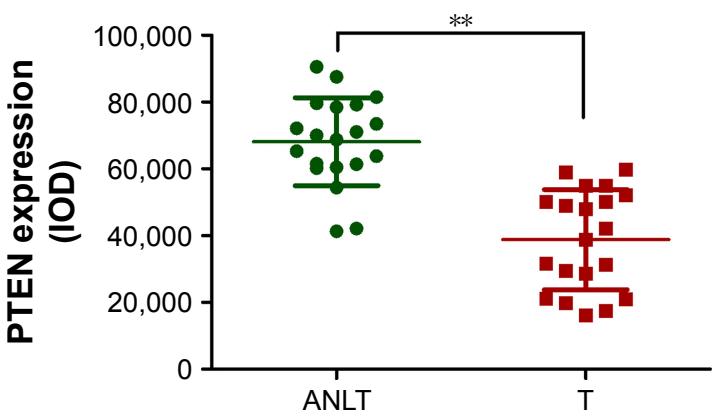

D

Patient 1 Patient 2 Patient 3 Patient 4

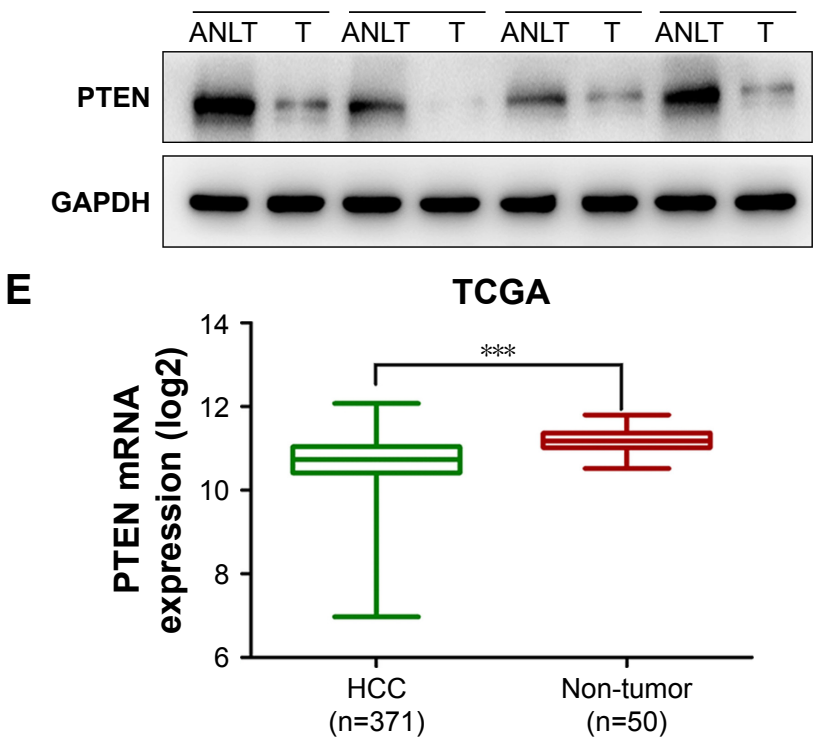

Figure S2 (Continued) 

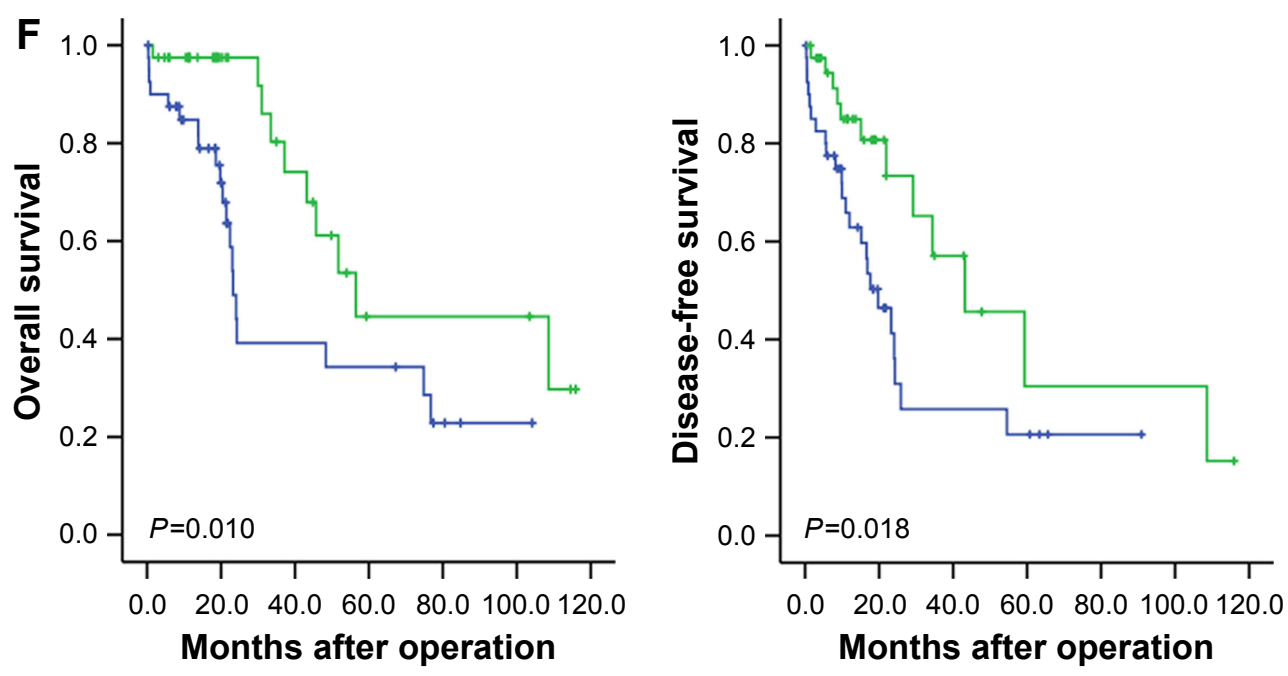

$\neg$ PTEN low expression $\backsim$ PTEN high expression

Figure S2 Clinical characteristics of PTEN in patients with HCC.

Notes: Representative IHC images (A) and statistical results (B) of PTEN expression in HCC tissues and ANLTs. Scale bars: (above) $100 \mu \mathrm{m}$, (below) $20 \mu \mathrm{m}$. (C) A negative relationship was found between miR-106b-5p and PTEN expression ( $R=-0.627, P=0.003)$. (D) Western blot analysis of PTEN protein level in four paired human HCC tissues and ANLTs. GAPDH was used as a loading control. (E) PTEN mRNA level in 37I human HCC tissues and 50 adjacent non-tumor liver tissues deposited in the TCGA database. (F) Kaplan-Meier analysis of overall survival and disease-free survival with low- or high-level PTEN expression. $* * P<0.01$, $* * * P<0.001$.

Abbreviations: PTEN, phosphatase and tensin homolog; IOD, integrated optical density; HCC, hepatocellular carcinoma; IHC, immunohistochemistry; ANLTs, adjacent non-tumor liver tissues; GAPDH, glyceraldehyde-3-phosphate dehydrogenase; TCGA, The Cancer Genome Atlas.

Table SI Association between PTEN expression and clinicopathological characteristics of patients with HCC

\begin{tabular}{|c|c|c|c|c|}
\hline \multirow{2}{*}{$\begin{array}{l}\text { Clinicopathological } \\
\text { variables }\end{array}$} & \multirow[t]{2}{*}{$\mathbf{n}$} & \multicolumn{2}{|l|}{ PTEN expression } & \multirow[t]{2}{*}{$P$-value ${ }^{a}$} \\
\hline & & $\begin{array}{l}\text { Low expression } \\
(\mathrm{N}=4 \mathrm{I})\end{array}$ & $\begin{array}{l}\text { High expression } \\
(\mathrm{N}=\mathbf{4 0})\end{array}$ & \\
\hline Sex & & & & 0.209 \\
\hline Male & 27 & 11 & 16 & \\
\hline Female & 54 & 30 & 24 & \\
\hline Age, years & & & & 0.733 \\
\hline$\leq 60$ & 43 & 21 & 22 & \\
\hline$>60$ & 38 & 20 & 18 & \\
\hline Liver cirrhosis & & & & 0.584 \\
\hline Absence & 28 & 13 & 15 & \\
\hline Presence & 53 & 28 & 25 & \\
\hline Tumor size, cm & & & & 0.003 \\
\hline$\leq 5$ & 64 & 27 & 37 & \\
\hline$>5$ & 17 & 14 & 3 & \\
\hline Microvascular invasion & & & & 0.032 \\
\hline Presence & 23 & 16 & 7 & \\
\hline Absence & 58 & 25 & 33 & \\
\hline Lymph node & & & & 0.735 \\
\hline Absence & 66 & 34 & 32 & \\
\hline Presence & 15 & 7 & 8 & \\
\hline Metastasis & & & & 0.963 \\
\hline Absence & 69 & 35 & 34 & \\
\hline Presence & 12 & 6 & 6 & \\
\hline Pathological stage & & & & 0.038 \\
\hline Early (I and II) & 68 & 31 & 37 & \\
\hline Late (III and IV) & 13 & 10 & 3 & \\
\hline
\end{tabular}

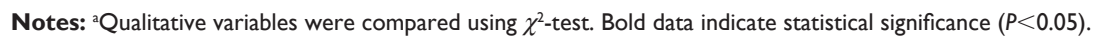

Abbreviations: PTEN, phosphatase and tensin homolog; HCC, hepatocellular carcinoma. 


\section{Publish your work in this journal}

OncoTargets and Therapy is an international, peer-reviewed, open access journal focusing on the pathological basis of all cancers, potential targets for therapy and treatment protocols employed to improve the management of cancer patients. The journal also focuses on the impact of management programs and new therapeutic agents and protocols on
Dovepress

patient perspectives such as quality of life, adherence and satisfaction. The manuscript management system is completely online and includes a very quick and fair peer-review system, which is all easy to use. Visit http://www.dovepress.com/testimonials.php to read real quotes from published authors.

Submit your manuscript here: http://www.dovepress.com/oncotargets-and-therapy-journal 